\title{
MOLECULAR DYNAMICS STUDY OF SOLID THIN-FILM THERMAL CONDUCTIVITY
}

\author{
Jennifer R. Lukes, Xin-Gang Liang ${ }^{*}$ and Chang-Lin Tien \\ Department of Mechanical Engineering \\ University of California \\ Berkeley, CA 94720
}

\section{ABSTRACT}

This study explores the feasibility of using the molecular dynamics computational technique to predict the thermal conductivity of solid thin films in the direction perpendicular to the film plane. The results show that thermal conductivity, as expected from thin-film experimental data and theoretical predictions, decreases as film thickness is reduced. In the large-size limit, this method yields thermal conductivities which asymptote to a value comparable to experimental data. The calculations modestly overpredict thermal conductivity, probably due to the use of a too-steep intermolecular potential. Most interestingly, an unusual wave effect is revealed for thin film thermal conductivity. This effect may be a manifestation of phonon wave interference analogous to the interference of light which determines the radiative properties of thin films.

It is also found that there are some temperature and computational domain size limitations on the applicability of molecular dynamics to the study of solid systems. A regime map is developed which delineates the conditions necessary for molecular dynamics to produce physically meaningful results. This work shows that molecular dynamics, applied under the correct conditions, is a viable tool for calculating the thermal conductivity of solid thin films. More generally, this work demonstrates the potential of molecular dynamics for ascertaining microscale thermophysical properties in more complex structures.

\section{NOMENCLATURE}

$\begin{array}{lll}a & = & \text { lattice constant } \\ E & = & \text { energy } \\ F & = & \text { total force on atom due to all atomic interactions } \\ f & = & \text { force between two atoms } \\ \hbar & = & \text { Planck's constant } / 2 \pi\end{array}$

$\begin{array}{lll}J_{Q} & = & \text { heat flux } \\ k & = & \text { thermal conductivity } \\ k_{B} & = & \text { Boltzmann's constant } \\ L & = & \text { simulation cell dimension } \\ M F P & = & \text { mean free path } \\ m & = & \text { atomic mass } \\ N & = & \text { number of atoms } \\ n & = & \text { equilibrium number of phonons } \\ p & = & \text { number of atomic planes } \\ R & = & \text { rescaling factor } \\ r & = & \text { position, displacement, or interatomic distance } \\ v & = & \text { velocity } \\ T & = & \text { temperature } \\ \Delta t & = & \text { time step } \\ V & = & \text { volume of atomic layer }\end{array}$

Greek $\varepsilon \quad=$ $\phi \quad=$

Lennard-Jones well depth parameter interaction potential Lennard-Jones equilibrium separation parameter frequency

Subscripts 1 smaller size or temperature half time step larger size or temperature applied calculated atom between atoms $\mathrm{i}$ and $\mathrm{j}$ layer Lennard-Jones

\footnotetext{
* Present address: Department of Engineering Mechanics, Tsinghua University, Beijing 100084, China
} 


$\begin{array}{lll}n & = & \text { new } \\ o & = & \text { old } \\ \text { sp } & = & \text { smallest periodic } \\ \text { sub } & = & \text { subtracted from rescaled velocity } \\ \text { trans } & = & \text { transition }\end{array}$

$\begin{array}{ll}\text { Superscripts } \\ D \quad= \\ F & =\end{array}$

self-diffusive

intermolecular

\section{INTRODUCTION}

Molecular dynamics (MD) is a computational method which simulates the real behavior of materials and calculates physical properties of these materials by simultaneously solving the equations of motion for a system of atoms interacting with a given potential. The computational work on anharmonic one-dimensional chains of atoms by Fermi, Pasta, and Ulam (Fermi et al., 1965) in the 1950s was the earliest contribution to the field of MD. This pioneering research was followed by other critical MD studies, including those of Alder and Wainwright (1960), Gibson et al. (1960), and Rahman (1964). A lack of sufficient computational power limited these and other early simulations to systems with a very small number of atoms.

In the past two decades, however, the number of MD studies has skyrocketed due to rapid developments in computer speed and memory. It is now possible, using parallel computation, to model systems on the order of a million atoms (Hoover et al., 1990). Still, the spatial domains treated by these "large" simulations remain very small. Even when periodic boundary conditions are used, constraints on size can complicate the calculation of bulk properties. The limitations of MD in simulating bulk materials can be turned to advantage for novel nanometer-scale materials such as buckyballs and buckytubes, highly nanoporous and ultrathin films, and quantum wires and dots. In particular, solid thin films are key components in integrated-circuit transistors and quantum-well lasers, and porous thin films of materials with favorable optical properties may play a critical role in the optical computing devices of the future (Matsumoto et al., 1995). Device performance in the above applications is very sensitive to operating temperature. For the best design of microdevices and thin-film materials, knowledge of thermophysical properties such as thermal conductivity is of paramount importance.

It is well known from measurements on thin films that such materials display markedly lower thermal conductivities than their bulk counterparts. Several approaches exist to predict the thin-film thermal conductivity for materials where heat conduction by lattice vibrations, or phonons, is dominant. These approaches include kinetic theory (Ziman, 1960), the Boltzmann transport theory-based equation of phonon radiative transfer (Majumdar, 1993), geometric analyses (Flik and Tien, 1990), and the Monte Carlo computational technique (Klitsner et al., 1988). Such methods, however, do not easily handle the nonuniformly distributed impurities, voids, cracks, dislocations, or complex geometries present in real films. Numerous papers on topics such as crack initiation and propagation (Hoagland et al., 1990), density of states of microcrystalline and glassy silicon nitride (Loong et al., 1995), and the effect of the intricate "cage" structure of clathrate hydrates on their thermal conductivity (Inoue et al., 1996), show that MD is well-suited for the study of nanoscale phenomena in solidphase materials. This approach provides a needed supplement to experimental measurements, which can be extremely difficult at such length scales. Additionally, MD can investigate behavior of materials at time scales which are often hard to access with experiments. Only time scales on the order of nanoseconds and shorter can be treated in a reasonable time by MD simulations, because the finite difference schemes used in such simulations require small time steps for energy conservation. Examples of short time scale MD studies in the literature include the transient heat conduction work of Volz et al. (1996) and the light-material interaction paper by Shibahara and Kotake (1998).

\section{MODEL OF ARGON-TYPE SOLID THIN FILMS}

Frequent topics of MD studies in the literature are fluid flow (Khare et al., 1997), phase change (Chokappa and Clancy, 1988), mass diffusion (Biggs and Agarwal, 1994), and the chemistry of fluids composed of large, complex molecules (Ryckaert and Bellemans, 1975). Typical properties calculated in these studies include energy, viscosity, and mass diffusion coefficient. Of all the materials simulated with MD in the above studies, argon-type fluid systems are overwhelmingly the most well-explored and characterized. Argon is a good choice for such simulations because the widely-accepted Lennard-Jones 12-6 (LJ) potential matches liquid-phase experimental data for argon reasonably well, and has meaningful physical constants as parameters. Additionally, its simple form, with only two-body terms, requires much less computation time than more complex potentials involving three-body and higher terms (Allen and Tildesley, 1987). A rigorous quantum-mechanical approach is currently not feasible for systems of more than a few atoms because such a method is too numerically intensive (Tersoff, 1988), although the efforts of many workers are currently directed toward this problem (Car and Parrinello, 1985; Car, 1996; Kotake, 1994).

Several MD studies have explored the thermal conductivity of bulk argon-type fluids. Nonequilibrium MD work by various groups, including Hoover and Ashurst (1975), Ciccotti and Tenenbaum (1980), Evans (1982), Tenenbaum et al. (1982), Massobrio and Ciccotti (1984), Müller-Plathe (1997) and Ikeshoji and Hafskjold (1994), demonstrates that the use of LJ potentials in MD simulations gives good agreement with experimental thermal conductivity data for bulk fluid argon. Fewer researchers have used MD to address the vibrational and thermophysical properties of solid materials. It is generally accepted that temperatures above the Debye temperature of a material are considered "high," or classical. The applicability of the classical LJ potential to solid bulk argon, then, may appear to be questionable, because argon is only solid up to $84 \mathrm{~K}$ and its Debye temperature is $80 \mathrm{~K}$. Experimental data (White and Woods, 1958) and other analyses (Keyes, 1959; Julian, 1965), however, show that the thermal conductivity of argon displays the classical inverse proportionality with temperature down to about $20 \mathrm{~K}$. As temperature decreases below this value, thermal conductivity first becomes larger than the classically predicted value due to the quantum mechanical freeze-out of Umklapp processes (Peierls, 1929), but then decreases with temperature due to the dominant effect of scattering from boundaries and imperfections. If these low-temperature regimes are avoided, MD using the classical LJ potential should yield results for bulk argon that are in line with experimental data.

Despite the technological importance of solid thin films in thermally sensitive applications, no MD calculation of the thermal conductivity of solid thin films as a function of size has been reported before, although related studies have been made. Kotake and Wakuri (1994) have shown for a two-dimensional solid system subjected to a constant flux that the resultant temperature gradients sharply increase as the system width is decreased, and recent work by Volz and Chen (1998) indicates that solid nanowires exhibit a strong reduction in 
thermal conductivity as compared to the bulk. Using MD in a threedimensional computational domain, the current paper explores thermal conductivity in a solid argon-type model system as the thickness in one dimension is reduced.

Thin films will exhibit different properties than bulk materials due to the existence of surfaces. The boundary conditions at these surfaces and the fraction of surface area relative to film volume will both influence the vibrational spectra for films, causing deviations from the bulk spectra. One may wonder, then, whether it is appropriate to apply the LJ potential, which models bulk fluids well, to solid thin films. Allen et al. (1969) and Dickey and Paskin (1970) used the LJ argon potential to perform MD simulations of nanoscale solid materials. The vibrational and specific heat behavior calculated from these simulations corresponds well qualitatively with experimental data (Novotny and Meincke, 1973) and with the results of other calculations (Burton, 1969; Wolf et al., 1995). For this reason, the present paper assumes that the $\mathrm{LJ}$ argon potential is applicable to thin film systems for qualitative studies of thermal conductivity.

Although argon is not a real thin-film material, it is the best choice for an initial thin-film thermal conductivity MD study. This is not only due to the fact that the results can be benchmarked against the large body of existing work on argon-type systems, but also because the argon model, as noted by Kristensen et al. (1974) and Wolf and Merkle (1992), should reveal fundamental thermal conductivity phenomena for a wide variety of materials in a computationally economical manner. Most importantly, the argon model is expected to span from the microscale regime to the bulk regime in a reasonable computational domain size due to its short phonon mean free paths. Unlike more technologically relevant materials which typically have long mean free paths, the argon model will give thermal conductivities which asymptote to the bulk value by simply adding a few more atomic layers in the "thin" direction. After establishing a firm foundation for pure thin films, the argon model can then be extended to thin-film systems with impurities, pores, defects, and other types of complicated structures. To get the most quantitatively accurate results for a given material, a potential specific to that material should be used, but for predicting qualitative trends, which is the aim of this paper, the argon model is a sensible choice.

\section{COMPUTATIONAL PROCEDURE}

Several example MD programs and subprograms are available in the literature and on the Internet. The majority of these only treat bulk systems at equilibrium, and no subroutines that calculate thin-film thermal conductivity by a direct nonequilibrium means could be found. It is possible to calculate thermal conductivity from an analysis of fluctuations of a system at equilibrium, but this method generally requires much more computation time than direct nonequilibrium simulations (Evans and Streett, 1978; Evans, 1979). To perform direct nonequilibrium MD calculations of solid thin-film thermal conductivity, a new MD program was constructed. This program is based on the equilibrium classical MD subroutines made available by Allen and Tildesley (1987), but substantial additions, revisions and modifications have been made to handle aperiodicity and to create a nonequilibrium condition.

The general approach of the program is to apply a constant flux to an argon-type solid system, to calculate the resulting temperature gradient, and to determine the thermal conductivity by a simple ratio of flux to temperature gradient. The reverse method, in which the film boundaries are kept at constant temperatures and the resultant flux is

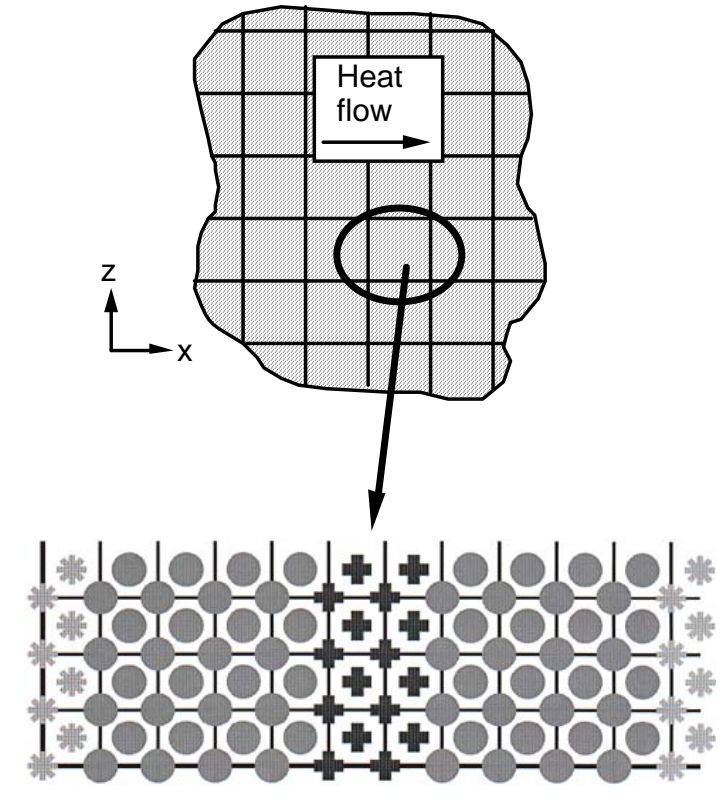
Fig. 1 Schematic of bulk thermal conductivity simulation
cell with sixteen regular atomic layers.

calculated, was initially tried. This was abandoned in favor of the current scheme due to the very slow convergence of the heat flux value. The execution of the program proceeds as follows. First, the simulation cell is constructed of face-centered cubic unit cells. Each unit cell contains four atoms and corresponds to two atomic layers of atoms. Each atom is assigned a type according to its spatial position: "hot," “cold," "regular," or "fixed." The configuration of the simulation cell depends upon whether bulk (Fig. 1) or perpendicular (Fig. 2) conductivity is to be calculated. In the figures, hot and cold regions are indicated by asterisks and plus signs, respectively, while the circles indicate regular atoms. Bulk materials are simulated by using periodic boundary conditions, in which the actual simulation cell of a small number of atoms is essentially repeated infinitely in all three coordinate directions. This method often yields values for physical properties surprisingly close to experimental results (Rahman, 1964; Verlet, 1967), but suppresses phonons in solids which have wavelengths larger than the simulation cell size (Allen and Tildesley, 1987).

For thin films, periodic boundary conditions are used in two coordinate directions. Fixed atoms, which remain stationary at their lattice positions for the entire simulation, are used in the third, "thin" direction to enforce an adiabatic boundary condition and to prevent evaporation of the film. These atoms are indicated by x marks in Fig. 2. Evaporation was found to be a problem in hotter regions of the film when a vacuum boundary condition was used. Following the example of Kotake and Wakuri (1994), four layers of fixed atoms are deemed sufficient to simulate an infinite wall due to the short range of the interatomic forces. All non-fixed atoms are given an initial temperature by choosing their velocities according to the MaxwellBoltzmann distribution at that temperature. After this, the program advances the difference equations of motion for a short equilibration period to allow a realistic thermodynamic state to be established.

The difference equations come from the widely-used "velocity Verlet” algorithm (Swope et al., 1982), 


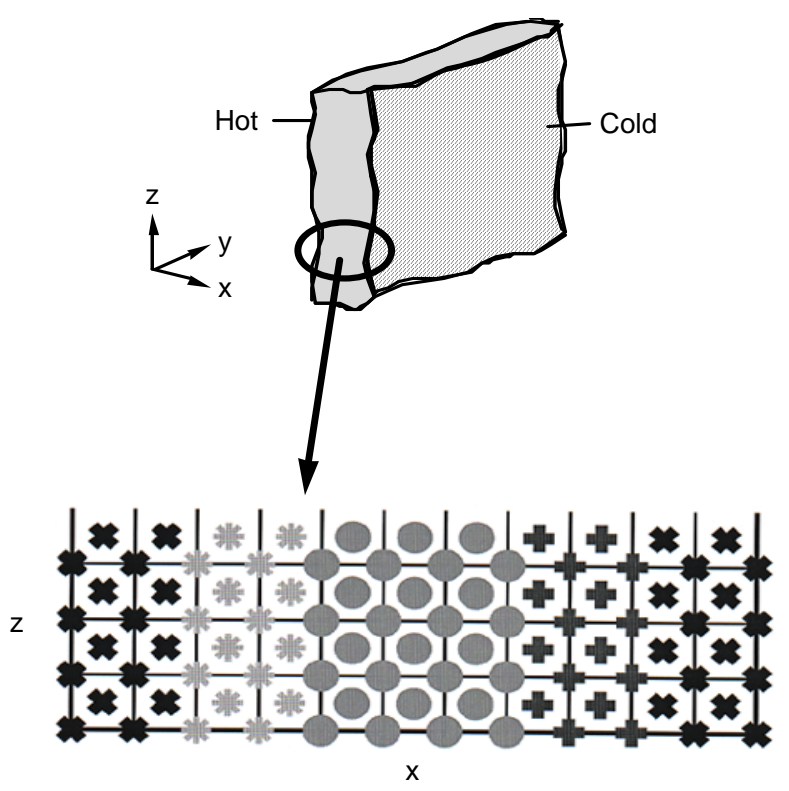

Fig. 2 Schematic of perpendicular thermal conductivity simulation cell with seven regular atomic layers.

$$
\begin{gathered}
r_{i_{n}}=r_{i_{0}}+v_{i_{o}} \Delta t+\frac{1}{2} \frac{F_{i_{0}}}{m_{i}}(\Delta t)^{2} \\
v_{i_{1 / 2}}=v_{i_{o}}+\frac{1}{2} \frac{F_{i_{0}}}{m_{i}} \Delta t \\
f_{i j_{n}}=-\frac{d \phi_{L J}\left(r_{i j_{n}}\right)}{d r_{i j_{n}}} \\
F_{i_{n}}=\sum_{j=1}^{N} f_{i j_{n}}, j \neq i \\
v_{i_{n}}=v_{i_{1 / 2}}+\frac{1}{2} \frac{F_{i_{n}}}{2} \Delta t
\end{gathered}
$$

In this algorithm, new atomic positions are calculated based on old positions, velocities, and forces (Eq. (1)). Velocities are calculated using a two-step scheme, in which old forces are used to advance old velocities to "half-step" velocities (Eq. (2)), new forces are calculated from the LJ potential using the new positions (Eqs. (3), (4)), and new velocities are found using the half-step velocities and new forces (Eq. (5)). The LJ potential is

$$
\phi_{L J}\left(r_{i j}\right)=4 \varepsilon\left\{\left(\frac{\sigma}{r_{i j}}\right)^{12}-\left(\frac{\sigma}{r_{i j}}\right)^{6}\right\}
$$

where $\varepsilon$ is the LJ well depth parameter and $\sigma$ is the equilibrium separation parameter. To keep computation time reasonable, the common convention of a cutoff radius is employed in calculating forces. This means that only the neighbors of an atom within a certain radius are included in the force calculations, since faraway atoms have a negligible contribution to the total force on a given particle. Such a convention should only be applied to systems in which atoms are subject to short-range forces (Allen and Tildesley, 1987).

After equilibration, a heat flux is imposed on the system by adding a fixed amount of energy to hot atoms and removing the same amount of energy from cold atoms at every time step. This induces heat flow in the $\mathrm{x}$-direction from the hot region to the cold region across the regular atoms, and is accomplished using the algorithm of Ikeshoji and Hafskjold (1994). In this algorithm, all energy is added/subtracted in the form of kinetic energy. Kinetic energies in the hot region are altered by scaling each hot atom's velocity by the same factor $\mathrm{R}$ and by subtracting a small velocity $\mathrm{v}_{\text {sub }}$ from this scaled velocity. The values of $R$ and $v_{\text {sub }}$ are chosen to conserve momentum and to add the desired amount of energy at each time step. A similar procedure is followed for the cold atoms, except kinetic energy is subtracted rather than added.

Once a steady state is reached, instantaneous temperatures in each regular atomic x-layer are time averaged for the duration of the simulation. Following the equipartition principle, the instantaneous temperature is calculated using the formula (Allen and Tildesley, 1987)

$$
T_{L}=\frac{m}{3 N_{L} k_{B}} \sum_{i=1}^{N_{L}} v_{i}^{2}
$$

where the squares of the magnitudes of the atomic velocities in a particular layer are summed. The gradient of the time-averaged temperatures in each layer is then taken, and the thermal conductivity is calculated by dividing the imposed heat flux by the negative of this gradient.

Use of Eq. (7) assumes that a local thermodynamic equilibrium (LTE) is established in each atomic layer and that there is a classical high-temperature heat capacity. Hafskjold and Ratkje (1995) explored the concept of LTE in nonequilibrium fluids by running several MD simulations on fluids subjected to mass and temperature gradients. They applied four different criteria for LTE to the results of these simulations, and concluded that the control volume size criterion of Tenenbaum et al. (1982) best satisfied the competing requirements of property uniformity (small control volume) and good statistics (large control volume) for nonequilibrium systems. This criterion is that control volume thickness for measurement of fluid properties should be roughly equal to the interatomic distance. Since this criterion was found to be valid for fluids, it should be even more likely to hold for atomic planes in solids because the increased density causes increased intermolecular interaction which aids energy transport between neighboring planes of the system. For these reasons, the criterion of Tenenbaum et al. was assumed to be valid so that Eq. (7) could be applied to each atomic plane. The development of linear temperature profiles in the calculations discussed below indicates that the assumption of LTE in each atomic layer is justified.

\section{RESULTS AND DISCUSSION}

\section{General comments about the simulations}

The simulations were run on DEC 3000 workstations and an AlphaServer 2000. Computation time was on the order of several hours, varying with the sizes of the systems considered. Two types of thermal conductivity simulation cases were run: bulk and perpendicular. Table 1 shows the fixed parameters used in most 
Table 1. Fixed simulation parameters

\begin{tabular}{c|c} 
Parameter & Value \\
\hline LJ well depth parameter [ $\varepsilon]$ & $1.67 \times 10^{-21} \mathrm{~J}$ \\
LJ equilibrium separation parameter [ $\sigma]$ & $3.4 \AA$ \\
Argon atomic mass [ m ] & $66.3 \times 10^{-27} \mathrm{~kg}$ \\
Boltzmann's constant [ k $\mathrm{B}]$ & $1.38 \times 10^{-23} \mathrm{~J} / \mathrm{K}$ \\
Lattice constant [ a ] & $5.31 \AA$ \\
Time step [ $\Delta \mathrm{t}]$ & $1 \mathrm{fs}$ \\
Applied dimensionless heat flux & 1.0 \\
Regular layers normal to the y- and z-directions ${ }^{2}$ & 8 \\
Layers in each hot or cold region ${ }^{3}$ & 4 \\
Layers in each fixed region & 4 \\
\hline
\end{tabular}

${ }^{1} 75 \mathrm{~K}$ runs use a dimensionless flux of 1.5

${ }^{2}$ For bulk simulations the number of regular $\mathrm{y}$ - and $\mathrm{z}$-layers is equal to the number of regular $\mathrm{x}$-layers

${ }^{3}$ Hot, cold, and fixed layers are always normal to the $\mathrm{x}$-direction

${ }^{4}$ Fixed layers are only used in the perpendicular case

simulations; any changes to these parameters for special case runs are noted. The nondimensionalizations used in the calculations are listed in Table 2. The configuration of the program is such that the initial temperature is specified, not the final average temperature of the lattice. In all runs, the temperature decreased over time from the initial value such that the final average lattice temperature was roughly half the initial temperature. This shows that half of the initial kinetic energy has been converted into potential energy, as dictated by equipartition. In the discussion that follows, the term "nominal temperature" is used for convenience to indicate one-half of the initial temperature. The true average lattice temperature was in all cases within a few degrees $\mathrm{K}$ of the nominal value.

For all simulations, several criteria were used to assess the validity of the generated data. These criteria include energy conservation, attainment of steady-state temperature profiles and heat flux distributions, and preservation of the lattice structure. Data from simulations which displayed large long-time energy drifts, nonlinear temperature profiles, significant differences in heat flux between regular atomic x-layers, or melting/evaporation characteristics were not used because in such cases the Fourier law, which is the basis of the program's thermal conductivity calculation, is not valid. To study the heat flux distributions, the

actual heat flux was calculated. The equations for heat flux are (Irving and Kirkwood, 1950)

$$
\begin{gathered}
\vec{J}_{Q}=\vec{J}_{Q}^{D}+\vec{J}_{Q}^{F} \\
\vec{J}_{Q}^{D}=\frac{1}{V} \sum_{i=1}^{N_{L}}\left(\frac{1}{2} m v_{i}^{2}+\frac{1}{2} \sum_{j=1}^{N} \phi\left(r_{i j}\right)\right) \vec{v}_{i}, j \neq i \\
\vec{J}_{Q}^{F}=-\frac{1}{2 V} \sum_{i=1}^{N_{L}} \sum_{j=1}^{N}\left(\vec{f}_{i j} \vec{v}_{i}\right) \vec{r}_{i j}, j \neq i
\end{gathered}
$$

The total flux (Eq. (8)) is the sum of the heat fluxes due to selfdiffusion (Eq. (9)) and intermolecular interaction (Eq. (10)). Heat flux was calculated in each $\mathrm{x}$-layer and was compared to the theoretical heat flux imposed by adding/subtracting energy in the source/sink regions. Since the program uses the theoretical value to calculate thermal conductivity, it is important that the calculated and the applied fluxes be as close as possible.

It was observed in several cases that simulations not run long enough to attain a steady state yielded calculated fluxes that were lower than the theoretical applied fluxes and thermal conductivities that were higher than longer-time values. The too-low calculated flux value at early times can be explained by the fact that the temperature gradient is still increasing then. The too-high thermal conductivity is an artifact resulting from the use of the theoretical value instead of the applied value in the program's thermal conductivity calculation. Both these discrepancies were observed to disappear as the number of time steps in the simulation increased. Calculated values of heat flux, mean temperature of the regular atoms, and thermal conductivity for the bulk and perpendicular thin-film runs are listed in Table 3. Calculated heat flux values were all within $4 \%$ of the theoretical applied flux. Correcting the thermal conductivity values in Table 3 by multiplying by the ratio $\mathrm{J}_{\text {Qcalc }} / \mathrm{J}_{\text {Qappl }}$ gave negligible changes in the values for all cases and did not change the shape or trend of any of the plots.

Momentum conservation, as found by Ikeshoji and Hafskjold (1994) in their bulk thermal conductivity simulations, was also found to hold for the present bulk simulations. In the thin-film simulations, however, total system momentum was not strictly conserved but fluctuated rapidly about zero. Physically, this could be viewed as a periodic accordion-like rippling of the solid between fixed walls. Since preliminary runs using a boundary condition in which wall atoms are not fixed but are tethered to their sites were found to conserve momentum, and since momentum non-conservation is absent in bulk simulations, the fixed wall boundary condition must be the cause of this effect. The fixed walls impart impulses to the atoms in the system in a manner analogous to billiard balls hitting a wall of infinite mass. The influence of non-zero momenta on thermal conductivity was explored by subtracting out the local x-plane velocities from the individual atomic velocities in the temperature calculations and using these modified temperatures to calculate the thermal conductivity. The differences in thermal conductivity between the modified and unmodified temperature simulations was very small, with differences only in the third significant digit or below. Based on this, it was concluded that the thin-film data from

\section{Table 2. Nondimensionalization equations}

\begin{tabular}{c|c}
\hline Quantity & Equation \\
\hline Length & $L^{*}=\frac{L}{\sigma}$ \\
Temperature & $T^{*}=\frac{k_{B} T}{\varepsilon}$ \\
Energy & $E^{*}=\frac{E}{\varepsilon}$ \\
Force & $F^{*}=\frac{F \sigma}{\varepsilon}$ \\
Time & $t^{*}=\frac{t}{\sigma} \sqrt{\frac{\varepsilon}{m}}$ \\
Velocity & $v^{*}=v \sqrt{\frac{m}{\varepsilon}}$ \\
Heat flux & $J_{Q}^{*}=J_{Q} \sigma^{3} \sqrt{\frac{m}{\varepsilon^{3}}}$ \\
Thermal conductivity & $k^{*}=k \frac{\sigma^{2}}{k_{B}} \sqrt{\frac{m}{\varepsilon}}$ \\
\hline
\end{tabular}


Table 3. Input parameters and calculated values

\begin{tabular}{|c|c|c|c|c|c|c|c|c|}
\hline \multicolumn{6}{|c|}{ Input Parameters } & \multicolumn{3}{|c|}{ Calculated Values } \\
\hline \multirow[t]{2}{*}{$\begin{array}{l}\text { Nominal lattice } \\
\text { temperature }^{1}\end{array}$} & \multirow[t]{2}{*}{ Configuration } & \multirow{2}{*}{$\begin{array}{l}\text { Number of } \\
\text { atoms or } \\
\text { layers }^{2}\end{array}$} & \multicolumn{3}{|c|}{$\begin{array}{c}\text { Number } \\
\text { of time steps }\end{array}$} & \multirow[t]{2}{*}{$\begin{array}{c}\text { Thermal } \\
\text { conductivity }^{3}\end{array}$} & \multirow[t]{2}{*}{$\begin{array}{l}\text { Mean lattice } \\
\text { temperature }^{1}\end{array}$} & \multirow[t]{2}{*}{$\begin{array}{l}\text { Heat } \\
\text { flux }^{3}\end{array}$} \\
\hline & & & $\mathrm{A}^{4}$ & $\mathrm{~B}^{5}$ & $\mathrm{C}^{6}$ & & & \\
\hline 35 & bulk & $\begin{array}{l}512 \\
576 \\
640 \\
704 \\
768\end{array}$ & $\begin{array}{c}3400 \\
5400 \\
7800 \\
10600 \\
13800\end{array}$ & $\begin{array}{c}8500 \\
13500 \\
19500 \\
26500 \\
34500\end{array}$ & $\begin{array}{c}25500 \\
40500 \\
58500 \\
79500 \\
103500\end{array}$ & $\begin{array}{l}28.3 \\
31.4 \\
34.3 \\
37.0 \\
40.6\end{array}$ & $\begin{array}{l}36.2 \\
37.1 \\
35.8 \\
36.0 \\
35.7\end{array}$ & $\begin{array}{l}0.962 \\
0.979 \\
0.992 \\
0.984 \\
0.993\end{array}$ \\
\hline 65 & bulk & $\begin{array}{c}96 \\
252 \\
512 \\
900 \\
1440 \\
2156 \\
3072 \\
\end{array}$ & $\begin{array}{c}3580 \\
5540 \\
7920 \\
10740 \\
14000 \\
17680 \\
21800\end{array}$ & $\begin{array}{c}18400 \\
28200 \\
40100 \\
54200 \\
70500 \\
88900 \\
109500\end{array}$ & $\begin{array}{c}35800 \\
55400 \\
79200 \\
107400 \\
140000 \\
176800 \\
218000\end{array}$ & $\begin{array}{l}21.2 \\
23.5 \\
25.2 \\
26.8 \\
27.4 \\
28.8 \\
30.7\end{array}$ & $\begin{array}{l}66.7 \\
71.3 \\
69.3 \\
69.2 \\
66.8 \\
66.7 \\
66.8 \\
\end{array}$ & $\begin{array}{l}1.031 \\
0.969 \\
0.996 \\
0.984 \\
0.983 \\
0.963 \\
0.984\end{array}$ \\
\hline 35 & perpendicular & $\begin{array}{l}3 \\
5 \\
7\end{array}$ & $\begin{array}{c}5400 \\
7800 \\
10600\end{array}$ & $\begin{array}{l}16200 \\
19500 \\
26500\end{array}$ & $\begin{array}{l}45000 \\
58000 \\
79500\end{array}$ & $\begin{array}{l}34.9 \\
37.7 \\
43.0\end{array}$ & $\begin{array}{l}35.7 \\
36.8 \\
35.7\end{array}$ & $\begin{array}{l}0.962 \\
0.970 \\
1.000\end{array}$ \\
\hline 50 & perpendicular & $\begin{array}{l}3 \\
5 \\
7 \\
9\end{array}$ & $\begin{array}{c}5400 \\
7800 \\
10600 \\
13800\end{array}$ & $\begin{array}{l}16200 \\
19500 \\
26500 \\
34500\end{array}$ & $\begin{array}{l}45000 \\
58500 \\
79500 \\
98080\end{array}$ & $\begin{array}{l}29.7 \\
30.7 \\
32.1 \\
38.1\end{array}$ & $\begin{array}{l}51.2 \\
53.1 \\
52.2 \\
51.5\end{array}$ & $\begin{array}{l}0.971 \\
0.986 \\
0.997 \\
1.001\end{array}$ \\
\hline 65 & perpendicular & $\begin{array}{c}3 \\
5 \\
7 \\
9 \\
11 \\
13\end{array}$ & $\begin{array}{c}6680 \\
9280 \\
12320 \\
15780 \\
19680 \\
24020\end{array}$ & $\begin{array}{c}33900 \\
46900 \\
62100 \\
79400 \\
98900 \\
120600\end{array}$ & $\begin{array}{c}66800 \\
92800 \\
123200 \\
207800 \\
246800 \\
290200\end{array}$ & $\begin{array}{l}24.5 \\
32.9 \\
35.4 \\
31.7 \\
35.3 \\
37.1\end{array}$ & $\begin{array}{l}69.7 \\
70.7 \\
68.8 \\
67.6 \\
66.5 \\
66.3\end{array}$ & $\begin{array}{l}0.977 \\
0.975 \\
0.998 \\
0.986 \\
0.983 \\
0.980\end{array}$ \\
\hline 75 & perpendicular & $\begin{array}{c}3 \\
5 \\
7 \\
9 \\
11 \\
13\end{array}$ & $\begin{array}{c}6680 \\
9280 \\
12320 \\
15780 \\
19680 \\
24020\end{array}$ & $\begin{array}{c}33900 \\
46900 \\
62100 \\
79400 \\
98900 \\
120600\end{array}$ & $\begin{array}{c}66800 \\
92800 \\
123200 \\
157800 \\
246800 \\
290200\end{array}$ & $\begin{array}{l}23.8 \\
28.8 \\
31.8 \\
32.8 \\
35.8 \\
38.3\end{array}$ & $\begin{array}{l}80.3 \\
80.0 \\
76.4 \\
75.3 \\
73.0 \\
71.7\end{array}$ & $\begin{array}{l}1.471 \\
1.471 \\
1.472 \\
1.455 \\
1.466 \\
1.471\end{array}$ \\
\hline
\end{tabular}

${ }^{1}$ Dimensions of $\mathrm{K}$

${ }^{2}$ Number of atoms for bulk simulations; number of layers for perpendicular simulations

${ }^{3}$ Dimensionless

${ }^{4}$ Time step at which flux is applied

${ }^{5}$ Time step at which time averaging begins

${ }^{6}$ Total time steps

unmodified temperature simulations was valid although momentum was not strictly conserved.

\section{Bulk thermal conductivity simulation}

Bulk thermal conductivity was calculated for simulation cells of various sizes at the nominal mean lattice temperatures of $35 \mathrm{~K}$ and 65 $\mathrm{K}$ to check the agreement of the simulation with experimental measurements on argon. The results are illustrated in Fig. 3. For the $35 \mathrm{~K}$ case, the calculated thermal conductivity is close to the experimental value. There are, however, two observations to be made. One is that the conductivity increases in a linear fashion as the number of atoms is increased, and the other is that values larger than the experimental values are found when the number of layers is larger than 640. An explanation of the second phenomenon may be that the simulation was run using the $0 \mathrm{~K}$ lattice constant, $5.31 \AA$. Typically MD simulations yield numbers that are smaller than the bulk values due to the exclusion of long-wavelength phonons, but using the $0 \mathrm{~K}$ density rather than the density that would exist at $35 \mathrm{~K}$ would cause increased intermolecular interaction and thus a higher thermal conductivity than expected.

The effect of using a more realistic density was explored for the $35 \mathrm{~K}, 704$ atom case at the same temperature by using a lattice constant of $5.337 \AA$, which corresponds to the $35 \mathrm{~K}$ experimentally measured density, instead of the lattice constant at $0 \mathrm{~K}$. As expected, the thermal conductivity for this case is lower than for the $0 \mathrm{~K}$ density case, with a dimensionless value of 35.4 as compared to 37.0. This is still, however, larger than the experimental value of 34.3. The fact that the simulated films are pure, while real films have impurities and other imperfections which reduce the thermal conductivity, may contribute to this difference. Another reason may be that although the lattice parameter was increased, the well depth and equilibrium separation parameters in the LJ potential were not adjusted to reflect this change. This causes the resting positions of the atoms to be different from the potential well minima, resulting in residual forces acting on the displaced atoms. Such forces would be reflected in the 


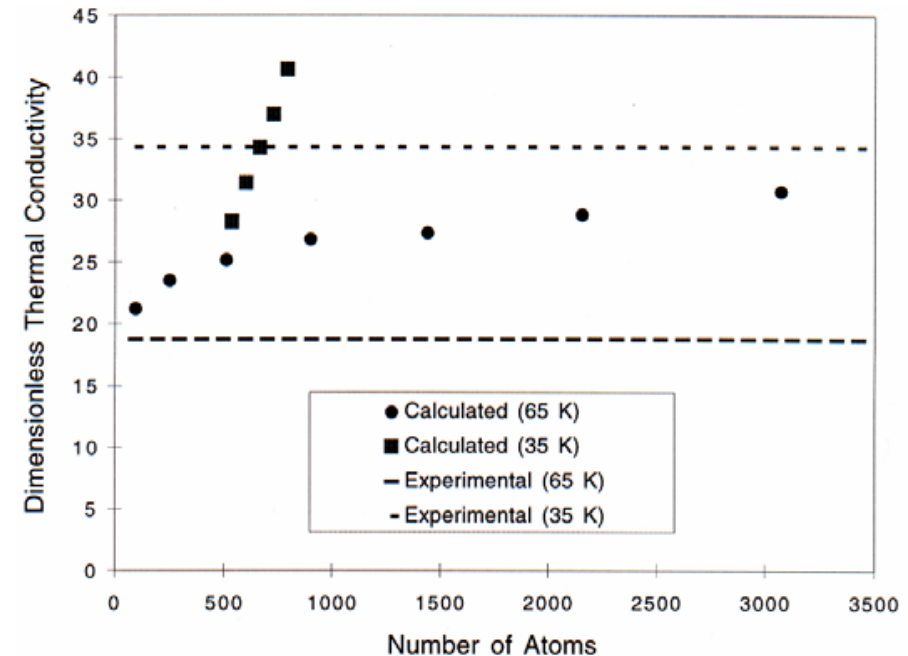

Fig. 3 Bulk thermal conductivity at $35 \mathrm{~K}$ and $65 \mathrm{~K}$ versus number of atoms. The experimental values are taken from Touloukian et al. (1970).

intermolecular contribution to the heat flux and may affect the thermal conductivity value.

Still another reason for the high values calculated in the simulation is that the Lennard-Jones potential may not be completely accurate for solid-phase materials. Berne et al. (1966) note that in the condensed phase, many-body effects tend to decrease the mean curvature of the potential well. This suggests that argon atoms are more loosely bound in actuality than what the LJ potential would predict. A too-steep potential means the "spring" binding atoms to one another is too tight, causing an artificially high intermolecular interaction and thus overpredicting the thermal conductivity. The idea that the intermolecular potential is the culprit is further bolstered by comparing the results of Ikeshoji and Hafskjold (1994), who applied a slightly modified LJ potential to an argon fluid, to the present results for LJ potential argon solids. The present computation uses the same configuration and calculation procedure on roughly the same number of argon atoms, yet gives a too-high thermal conductivity while Ikeshoji and Hafskjold's result agrees with experimental data within 1 - 2\%. Although the calculated bulk thermal conductivities are in some cases larger than the experimental data, they seem reasonable based on the discussion above.

The other phenomenon observed above for the $35 \mathrm{~K}$ curve, an increase of bulk thermal conductivity as the number of atoms is increased, has also been found in an equilibrium MD simulation performed by Volz and Chen (1998). This behavior indicates that the bulk-configuration simulation cell of Fig. 1, which is the next to largest size explored, is not large enough to well-represent the true bulk properties of solid argon at $35 \mathrm{~K}$. According to Allen and Tildesley (1987), use of periodic boundary conditions with a simulation cell of this size should be large enough to produce bulklike behavior in fluids, but the results clearly show that the same assumption is not true for solids. True bulk-like behavior would be indicated by little or no size dependence in the calculated thermal conductivity value. As mentioned above, long-wavelength phonons are artificially excluded in MD "bulk" simulations because spatial fluctuations with wavelengths larger than the simulation cell size

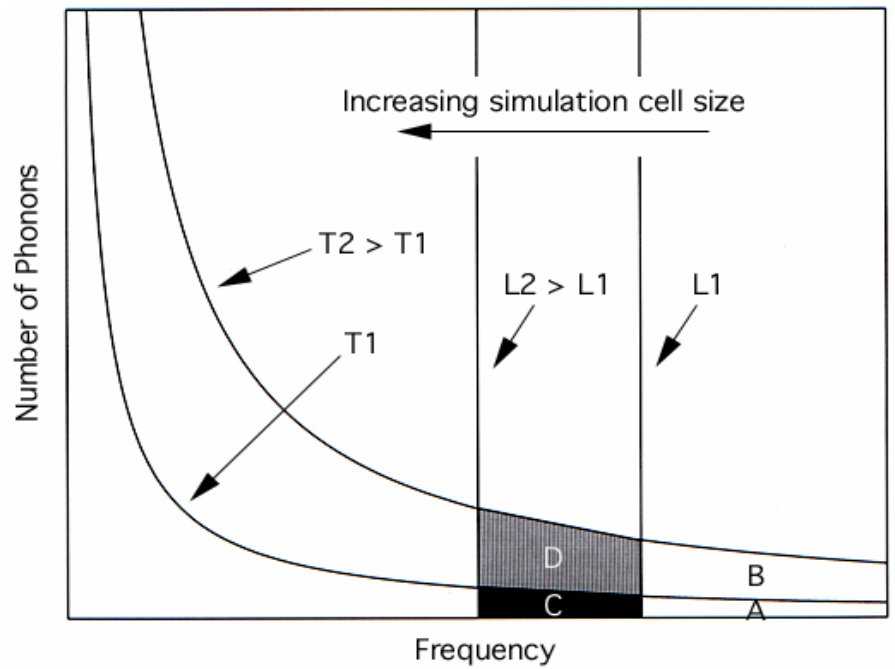

Fig. 4 Bose-Einstein distribution for phonons.

cannot be captured (Hansen and McDonald, 1986). To replicate bulk behavior, the results suggest that the MD simulation should be run using a larger number of atoms. This would cause an undesirable increase in simulation time.

A better alternative would be to run the simulation at a higher temperature. This reasoning is illustrated in Fig. 4, which shows the Bose-Einstein distribution for phonons at two different temperatures. This distribution,

$$
n=\frac{1}{\exp \left(\frac{\hbar \omega}{k_{B} T}\right)-1}
$$

gives the equilibrium number of phonons present as a function of temperature and frequency. The vertical lines on the figure indicate minimum phonon frequencies allowed by the size of the simulation cell for two different cases. At a given size, no phonons with frequencies to the left of this line can exist. For example, region A indicates the number of phonons present at temperature $\mathrm{T} 1$ and simulation cell size L1. As size increases from L1 to L2 while remaining at $\mathrm{T} 1$, the maximum phonon wavelength allowed increases. The minimum frequency thus decreases and the phonons in $\mathrm{C}$ are now allowed, so that the total number of phonons is represented by $\mathrm{A}+\mathrm{C}$. In the limit of zero frequency or infinite size, all phonons are allowed by the simulation cell. Raising the temperature from $\mathrm{T} 1$ to $\mathrm{T} 2$ while remaining at size L1 shifts the distribution higher, effectively moving the minimum frequency closer to the steeper part of the curve so that a greater fraction of the total area under the curve is occupied. This means that the higher-temperature case is "closer" to bulk behavior. The additional phonons added in this case are in B, so that the total number of phonons is $\mathrm{A}+\mathrm{B}$. Increasing both size and temperature yields $\mathrm{A}+\mathrm{B}+\mathrm{C}+\mathrm{D}$. Ideally, larger sizes and higher temperatures should be used to best model bulk behavior, but using higher temperatures is the most computationally economical choice.

The phonon distribution of Eq. (11) displays an exponential dependence on frequency in the high-frequency limit, 


$$
n \sim \exp \left(-\frac{\hbar \omega}{k_{B} T}\right), \quad \frac{\hbar \omega}{k_{B} T}>>1
$$

and has an inverse proportionality at low frequencies,

$$
n \sim \frac{k_{B} T}{\hbar \omega}, \quad \frac{\hbar \omega}{k_{B} T}<1
$$

The transition to the desired bulk-like low frequency regime should occur near a dimensionless parameter value of 1 . This is exactly the same as the so-called Wien's law for phonons and is analogous to the result for photons. Substituting for frequency and using the $10 \mathrm{MPa}$, $90 \mathrm{~K}$ speed of sound in fluid argon because it was the highest tabulated value for any form of argon found in the CRC Handbook (1996), it can be shown that the Wien's law for phonons requires

$$
\left(p_{\text {sp }} T\right)_{\text {trans }} \sim 153
$$

for transition, where $\mathrm{p}_{\mathrm{sp}}$ is the number of atomic planes along the periodic direction of the simulation cell which has the shortest length and 153 is in degrees Kelvin. A $\mathrm{p}_{\mathrm{sp}} \mathrm{T}$ product as much larger than 153 as possible should be used for an MD simulation, because this will ensure that the simulation is run in the desired bulk-like regime.

To investigate the effect of higher temperature on bulk thermal conductivity, another set of simulations was run at $65 \mathrm{~K}$ (Fig. 3). In this case, the size of the simulation cell was changed not only by increasing the number of regular x-layers as in the $35 \mathrm{~K}$ case, but also by adding $\mathrm{y}$ - and z-layers so that the number of $\mathrm{y}$ - and $\mathrm{z}$-layers equaled the number of regular x-layers. Similarly to the $35 \mathrm{~K}$ case, the $65 \mathrm{~K}$ case shows an increase in thermal conductivity with simulation cell size. The important difference between the two curves is that the $65 \mathrm{~K}$ data appear to asymptote toward a dimensionless value of around 32, while the $35 \mathrm{~K}$ data display a linear increase which shows no signs of tapering off. This result is strong proof that the 65 $\mathrm{K}$ simulation is much closer to the bulk regime because changing the computational cell size has only a weak effect on the results. A simulation cell size of 512 atoms, which corresponds to 4 unit cells or 8 atomic planes in each coordinate direction, yields thermal conductivity values not much smaller than simulations with a significantly larger number of atoms. For computational efficiency, 8 planes in a particular periodic coordinate direction is assumed enough to capture the essential physics for a system with a temperature equal to or greater than $65 \mathrm{~K}$. In the perpendicular thermal conductivity simulations of the following section, then, 8 planes were used in the periodic $y$ - and z-directions to represent "infinite" length in those dimensions.

\section{Perpendicular thermal conductivity simulation}

Perpendicular thermal conductivity versus film thickness is illustrated in Fig. 5 for simulations run at nominal mean lattice temperatures of $65 \mathrm{~K}$ and $75 \mathrm{~K}$. Results from lower-temperature simulations are also shown as individual data points, and will be discussed later. In the simulations, film thickness was varied by adding/subtracting atomic x-layers to/from the computational cell. Experimental bulk thermal conductivity data and curves produced from Majumdar's equation of phonon radiative transfer, or "EPRT" (Majumdar, 1993) are shown for comparison to the $65 \mathrm{~K}$ and $75 \mathrm{~K}$ calculated values. The EPRT approach is based on solving the

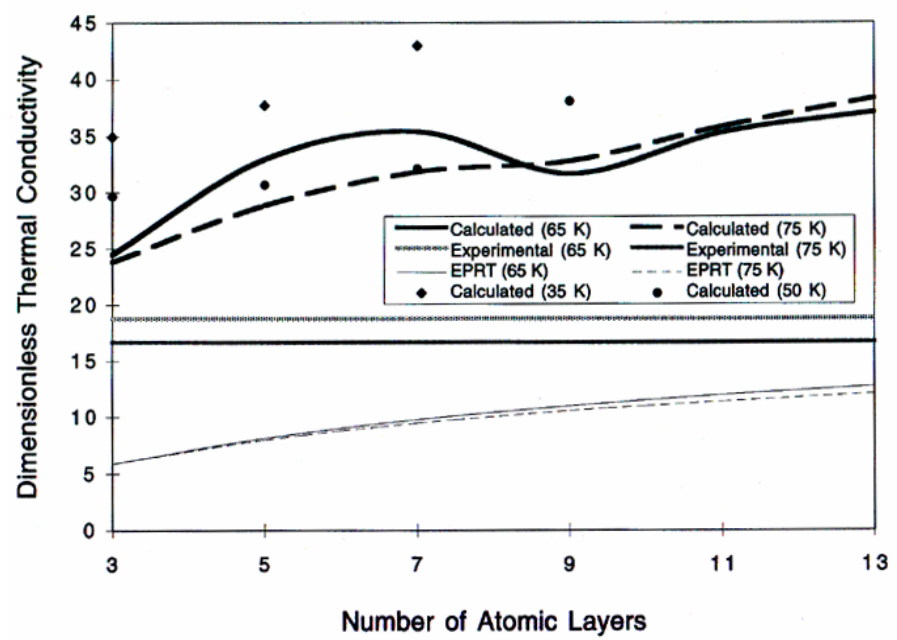

Fig. 5 Perpendicular thermal conductivity at $65 \mathrm{~K}$ and $75 \mathrm{~K}$ versus number of $\mathrm{x}$-layers. The experimental values are taken from Touloukian et al. (1970). EPRT values were calculated using the equation of phonon radiative transfer (Majumdar, 1993). Diamonds and circles indicate lowertemperature simulations which have questionable validity.

Boltzmann transport equation for phonons in dielectric thin films. Solid argon properties found in Dobbs and Jones (1957), the speed of sound mentioned above, and the mean free path equation of Dugdale and MacDonald (1954) were used for the EPRT calculations. Figure 6 shows a representative temperature profile generated from the $65 \mathrm{~K}, 5$ layer simulation. In this figure, the fixed atoms on either side are at 0 $\mathrm{K}$ and the source (hot) and sink (cold) regions display the expected parabolic temperature profile. The temperatures of the regular atoms are well fitted by a straight line whose slope was used to determine the thermal conductivity.

The $75 \mathrm{~K}$ calculated thermal conductivity in Fig. 5 increases fairly smoothly as the number of layers is increased. The same trend was found by Kotake and Wakuri (1994) for the thermal conductivity of a two-dimensional argon-type system whose width was varied, and can be seen also in both EPRT curves, which approach the experimental bulk values nicely as size is increased. The calculated curves are higher than the EPRT curves and their large-size limiting values are larger than the experimental values by a factor of two. This is not a surprising result since the bulk calculations of the previous section were also found to be higher than experimental data. As discussed before, the reason for this is probably that the $\mathrm{LJ}$ potential is too stiff to use for solids.

Also, as the number of layers increases beyond 9, the thermal conductivity curve takes a slight upward bend and attains values higher than for the $65 \mathrm{~K}$ curve. The $75 \mathrm{~K}$ results are expected to be lower than the $65 \mathrm{~K}$ results due to the greater anharmonicity of vibration at the higher temperatures. As mentioned above, overly high thermal conductivities are commonly obtained when simulations are not run long enough. The $65 \mathrm{~K}$ and $75 \mathrm{~K}$ simulations were in almost all cases run for the same number of time steps, so a too-short simulation time does not seem to be the reason. A more likely reason is that as the number of layers increases, the mean lattice temperature decreases (Table 3). Thermal conductivity in general increases with decreasing temperature, so this could very well be the cause of the 


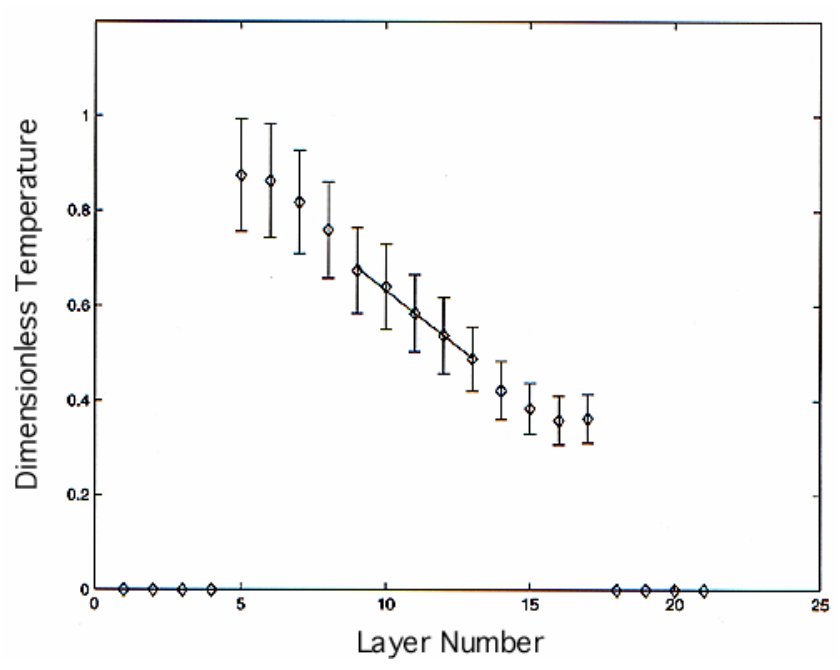

Fig. 6 Temperature in each atomic layer of the thin film for the 5 layer, $65 \mathrm{~K}$ case. The error bars are one standard deviation of the instantaneous temperature values.

high values. The "75 K" simulation is actually comprised of an almost 9 degree range of temperatures, while the " $65 \mathrm{~K}$ " simulation displays much less temperature variation. This may be due to a larger noise component in the instantaneous temperature values at $75 \mathrm{~K}$. It might also be caused the larger dimensionless heat flux of 1.5, which was used to try to combat the noise so that a discernible gradient could be established. Future work on higher-temperature systems should use some means to assure a suitably "constant" average lattice temperature so that effects of temperature variation do not obscure the effects of size.

A more surprising finding is that the calculated thin-film values are larger than the calculated bulk values! It is unclear at present why this is the case, but a potential well overstiffness argument may again be applicable here. The boundary conditions for the thin film case are fixed walls whose component atoms are not permitted to move. Considering interactions between atoms in a three-dimensional massspring context, one can visualize that a mobile atom moving toward another mobile atom should experience more "give" than a mobile atom approaching a fixed atom. This would happen because the mobile atom being approached is free to move sideways or in some other direction to minimize the mutual repulsion while the fixed atom is not. The effect of this would be that the mobile/fixed spring appears stiffer than the mobile/mobile spring. The overly stiff spring would result in overprediction of thermal conductivity, as discussed above. To get a more definitive answer to this question, the effect of wall atom stiffness should be explored by allowing wall atoms to move while tethering them to defined lattice sites with different spring strengths.

The most interesting result of the thin-film thermal conductivity calculation was found for the $65 \mathrm{~K}$ simulation. An unusual wave effect for this case can be seen in Fig. 5. As the number of layers is increased from 3 to 7 , the thermal conductivity increases monotonically. At the 9 layer point, the curve takes a sudden downturn, and by the 11 layer point, the curve has increased back up to the 7 layer value. For larger sizes the curve tapers off. Within the limitations of the simulation, these results are believed not to be a fluke because they were reproducible for three separate sets of lattice initializations. Unlike the $75 \mathrm{~K}$ case, this behavior cannot be attributed to temperature effects because the 9 and 11 layer simulation temperatures are lower than those of the smaller simulations. Thermal conductivity would be expected to continue increasing for this reason, not to display a down dip and subsequent upturn. It could be that the fixed wall atom boundary condition, which is believed to at least partially be the cause of the other phenomena discussed above, could create spurious effects in the data. This does not explain , however, why the $75 \mathrm{~K}$ curve shows a fairly smooth increase while the $65 \mathrm{~K}$ curve does not.

An intriguing possibility is that the MD simulation has revealed phonon wave effects on thermal conductivity. Using the transfer matrix method for acoustic waves, Chen (1998) found such effects for sufficiently thin films. The results of the MD simulation may provide an independent confirmation of Chen's results. The $65 \mathrm{~K}$ curve in Fig. 5 resembles the thermal conductivity versus film thickness curve in Chen's paper in that there is a steeper increase for smaller thicknesses followed by the above-mentioned tapering off for larger thicknesses. The definite peak and valley of the $65 \mathrm{~K}$ curve at 7 and 9 layers, however, is not visible in Chen's curve. One reason for this may be that at $65 \mathrm{~K}$, the coherence length of the blackbody "phonon radiation" is long enough to be on the order of or greater than the simulation cell sizes considered in the MD simulation. Long coherence lengths relative to the spatial scale of observation are required for pronounced wave interference effects to occur. From Mehta's work (1963) on the effective coherence time and bandwidth of blackbody photon radiation, it can be seen that coherence time, and thus coherence length, is inversely proportional to temperature. At 75 $\mathrm{K}$ there are no clear peaks or valleys, probably because raising the temperature causes an decrease in the effective coherence length which weakens interference effects. To verify that MD simulations can detect phonon wave effects, several sets of simulations run at temperatures lower than $65 \mathrm{~K}$ should be made. These simulations should show even stronger oscillations than for the $65 \mathrm{~K}$ case.

The effect of lower temperatures on perpendicular thermal conductivity was explored using a simulation cell with the same lateral dimensions (8 layers) as the $65 \mathrm{~K}$ and $75 \mathrm{~K}$ cases. Figure 5 shows, as individual data points, the results of runs at $50 \mathrm{~K}$ and $35 \mathrm{~K}$ in addition to the $65 \mathrm{~K}$ and $75 \mathrm{~K}$ results discussed above. It can be seen that the $50 \mathrm{~K}$ and $35 \mathrm{~K}$ curves, in contrast to those at higher temperatures, display a strange upward concavity. It is postulated here that the reason for the unusual behavior at lower temperatures which disappears in the $65 \mathrm{~K}$ temperature run is a transition to the steeper, bulk-like regime where an adequate number of longwavelength phonons is allowed by the simulation cell lateral size. Recall from the discussion in the previous section and from Fig. 3 that $35 \mathrm{~K}$ "bulk" simulations using 8 layers in the shortest periodic directions show distinctly non-bulk, incorrect behavior because the thermal conductivity increases sharply and steadily with simulation cell size. Similarly, results from perpendicular conductivity simulations at $35 \mathrm{~K}$ which use only 8 layers in the "infinite" $\mathrm{y}$ - and $\mathrm{z}$ directions will also be incorrect. It is concluded, then, that curves which look like the $35 \mathrm{~K}$ curve contain unphysical artifacts and data from these curves should not be trusted. Curves displaying the expected behavior, such as those at the two higher temperatures, are assumed to have enough length in the lateral dimensions to produce meaningful results. The $50 \mathrm{~K}$ simulation, then, also suffers from lateral dimensions that are too small. To avoid obscuring any wave effect behavior, the data indicates that larger simulation cells must be used for colder temperature simulations. 
Based on the fact that there were 8 atomic planes in both $\mathrm{y}$ and $\mathrm{z}$ directions for the $65 \mathrm{~K}$ and $75 \mathrm{~K}$ perpendicular runs, and using the 65 $\mathrm{K}$ run as a conservative base case for comparison, an improvement to the transition criterion of Eq. (14) is suggested:

$$
\left(p_{\text {sp }} T\right)_{\text {trans }} \sim 520
$$

Here $\mathrm{p}_{\mathrm{sp}}$ is the number of atomic layers in the periodic direction with the smallest length and 520 is in degrees Kelvin. In terms of simulation cell size, Eq. (15) becomes

$$
\left(L_{\text {sp }} T\right)_{\text {trans }} \sim 138
$$

where $L_{s p}$ is the smallest periodic simulation cell dimension and 138 is in units of nm-K. Note that Eqs. (15) and (16) are only valid for argon, since argon parameters were used in their derivation.

The importance of Eqs. (15) and (16) are that they are guidelines to use in MD simulations. When the $\mathrm{p}_{\mathrm{sp}} \mathrm{T}$ product is greater than 520 or the $\mathrm{L}_{\mathrm{sp}} \mathrm{T}$ product is greater than 138 , artifacts from the periodic boundary condition should no longer be important because enough long-wavelength phonons will be allowed in the periodic dimensions of the simulation cell to give results with physical meaning. Of course more data is needed to best pinpoint the correct transition value, but the results clearly indicate that high-temperature simulations produce physical results for reasonably-sized simulation cells while lowtemperature simulations do not. MD simulations are quite timeconsuming, so the use of Eqs. (15) and (16) will help to minimize trial and error for those performing such simulations. Figure 7 is a regime map which illustrates which temperatures and sizes should be avoided and which should be used in MD simulations. In this figure, a dimensionless form of the $\mathrm{L}_{\mathrm{sp}}$ at transition is shown as a function of temperature. At a given temperature, simulation cells with a smallest periodic dimension below this transition value give unphysical results due to the cutoff of long-wavelength phonons. This is indicated by the shaded regime in Fig. 7. Also shown in Fig. 7 is a plot of the dimensionless mean free path, which comes from values calculated from solid argon parameters given by Dobbs and Jones (1957). The purpose of the mean free path plot is to illustrate that at high enough temperatures, LJ argon-model MD simulations should be able to span from the microscale to bulk thermal conductivity regimes using reasonably small computational domains. The $65 \mathrm{~K}$ and $75 \mathrm{~K}$ curves in Fig. 5 suggest that this is indeed the case, indicating that MD using the argon model is a viable method for determining the perpendicular thermal conductivity of solid thin films.

\section{CONCLUDING REMARKS}

This paper explored the thermal conductivity of solid systems in both bulk and thin-film configurations using the molecular dynamics computational technique (MD). It was found, as expected from previous work in the literature, that MD using an argon model yields results close to experimental data for bulk materials and predicts increased thermal conductivity for thin film materials as number of atomic layers is increased. Modest overprediction of thermal conductivity by the simulations was attributed to overstiffness of the LJ potential in application to solids. Additionally, the simulations revealed an unusual thermal conductivity wave effect which may be a manifestation of phonon wave interference. A strange result found for MD simulations run at low temperatures was attributed to the cutoff of long-wavelength phonons for these conditions. Using the calculated

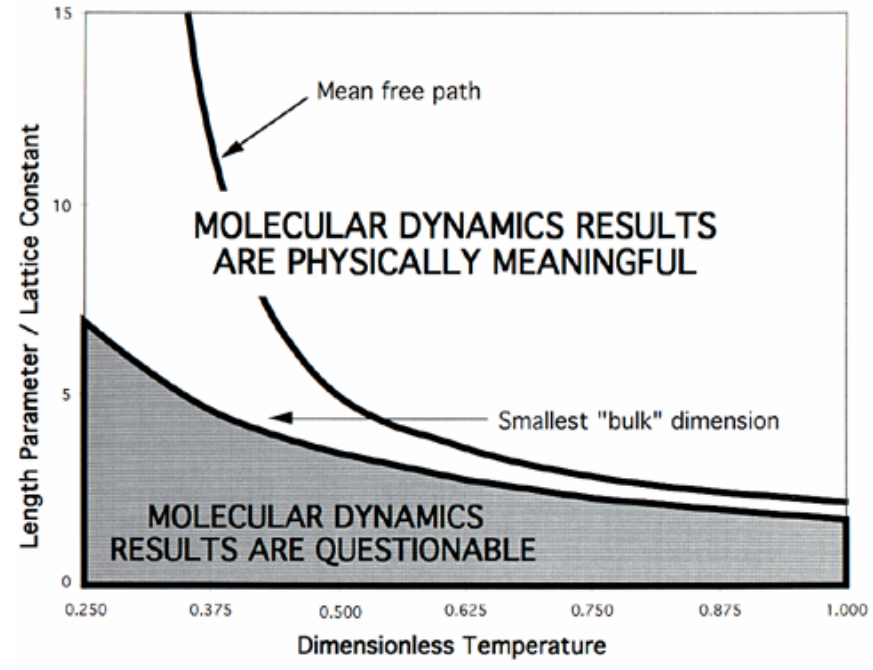

Fig. 7 Regime map illustrating the temperature ranges and dimensionless length scales for which molecular dynamics using the argon model yields physically meaningful results. Also shown for comparison is a plot of the dimensionless mean free path.

data, equations were developed and a map was constructed which delineate regimes where $\mathrm{MD}$ simulations produce physically reasonable results and where they provide results of questionable validity. This work has shown that MD, when applied at the right combinations of temperature and simulation cell size, is a powerful tool for predicting the thermal behavior of solid thin films. Future work should be done to apply this versatile, conceptually simple technique to microscale problems where other experimental and analytical approaches are difficult. MD is especially suited to study the thermophysical properties of more complex microstructures such as doped and nanoporous thin films and materials with voids, cracks, dislocations, or other complex geometries.

\section{ACKNOWLEDGMENTS}

The authors gratefully acknowledge the financial support of the National Science Foundation and wish to thank Professor Arun Majumdar for suggesting the exploration of wave effects, Professor Koji Takahashi for providing initial guidance on molecular dynamics, Dr. Sebastian Volz for giving helpful comments and suggestions, and Jeffrey Persch for optimizing the computer program.

\section{REFERENCES}

Alder, B. J. and Wainwright, T. E., 1960, "Studies in Molecular Dynamics. II. Behavior of a Small Number of Elastic Spheres," Journal of Chemical Physics, Vol. 33, pp. 1439-1451.

Allen, M. P. and Tildesley, D. J., 1987, Computer Simulation of Liquids, Clarendon Press, Oxford.

Allen, R. E., de Wette, F. W. and Rahman, A., 1969, "Calculation of Dynamical Surface Properties of Noble-Gas Crystals. II. Molecular Dynamics,” Physical Review, Vol. 179, pp. 887-892.

Berne, A., Boato, G. and de Paz, M., 1966, "Experiments on Solid Argon,” Il Nuovo Cimento, Vol. 46 B, pp. 182-208. 
Biggs, M. and Agarwal, P., 1994, "Mass Diffusion of Diatomic Fluids in Random Micropore Spaces Using Equilibrium Molecular Dynamics,” Physical Review E, Vol. 49, pp. 531-537.

Burton, J. J., 1969, "Anomalous Heat Capacity of Spherical Clusters of Atoms,” Chemical Physics Letters, Vol. 13, pp. 594-596.

Car, R., 1996, "Modeling Materials by Ab-Initio Molecular Dynamics," Quantum Theory of Real Materials, J. R. Chelikowsky and S. G. Louie, eds., Kluwer Academic Publishers, Norwell, pp. 2337.

Car, R. and Parrinello, M., 1985, "Unified Approach for Molecular Dynamics and Density-Functional Theory,” Physical Review Letters, Vol. 55, pp. 2471-2474.

Chen, G., 1998, "Phonon Wave Effects on Heat Conduction in Thin Films," Proceedings of the 7th AIAA/ASME Joint Thermophysics and Heat Transfer Conference, Vol. 3, pp. 205-213, and submitted to Journal of Heat Transfer.

Chokappa, D. K. and Clancy, P., 1988, "The Influence of an Interface in the Promotion of Melting," Molecular Physics, Vol. 65, pp. 97-107.

Ciccotti, G. and Tenenbaum, A., 1980, "Canonical Ensemble and Nonequilibrium States by Molecular Dynamics,” Journal of Statistical Physics, Vol. 23, pp. 767-772.

CRC Handbook of Chemistry and Physics, 1996, CRC Press, Boca Raton.

Dickey, J. M. and Paskin, A., 1970, "Size and Surface Effects on the Phonon Properties of Small Particles," Physical Review B, Vol. 1, pp. 851-857.

Dobbs, E. R. and Jones, G. O., 1957, "Theory and Properties of Solid Argon,” Reports on Progress in Physics, Vol. 20, pp. 516-564.

Dugdale, J. S. and MacDonald, D. K. C., 1954, "Vibrational Anharmonicity and Lattice Thermal Properties," Physical Review, Vol. 96, pp. 57-62.

Evans, D. J., 1979, “The Frequency Dependent Shear Viscosity of Methane,” Molecular Physics, Vol. 37, pp. 1745-1754.

Evans, D. J., 1982, "Homogeneous NEMD Algorithm for Thermal Conductivity - Application of Non-Canonical Linear Response Theory," Physics Letters, Vol. 91A, pp. 457-460.

Evans, D. J. and Streett, W. B., 1978, "Transport Properties of Homonuclear Diatomics. II. Dense Fluids,” Molecular Physics, Vol. 36, pp. 161-176.

Fermi, E., Pasta, J. R. and Ulam, S., 1965, Collected Papers of Enrico Fermi, University of Chicago Press, Chicago.

Flik, M. I. and Tien, C. L., 1990, "Size Effect on the Thermal Conductivity of High-Tc Thin-Film Superconductors," Journal of Heat Transfer, Vol. 112, pp. 872-881.

Gibson, J. B., Goland, A. N., Milgram, M. and Vineyard, G. H., 1960, “Dynamics of Radiation Damage,” Physical Review, Vol. 120, pp. 1229-1253.

Hafskjold, B. and Ratkje, S. K., 1995, "Criteria for Local Equilibrium in a System with Transport of Heat and Mass," Journal of Statistical Physics, Vol. 78, pp. 463-494.

Hansen, J.-P. and McDonald, I. R., 1986, Theory of Simple Liquids, Academic Press, London.

Hoagland, R. G., Daw, M. S., Foiles, S. M. and Baskes, M. I., 1990, "An Atomic Model of Crack Tip Deformation in Aluminum Using an Embedded Atom Potential," Journal of Materials Research, Vol. 5, pp. 313-324.

Hoover, W. G. and Ashurst, W. T., 1975, "Nonequilibrium Molecular Dynamics," Theoretical Chemistry: Advances and Perspectives, H. Eyring and D. Henderson, eds., Academic Press, New York, pp. 1-51.
Hoover, W. G., de Groot, A. J., Hoover, C. G., Stowers, I. F., Kawai, T., Holian, B. L., Boku, T., Ihara, S. and Belak, J., 1990, "Large-Scale Elastic-Plastic Indentation Simulations via Nonequilibrium Molecular Dynamics,” Physical Review A, Vol. 42, pp. 5844-5853.

Ikeshoji, T. and Hafskjold, B., 1994, "Non-equilibrium Molecular Dynamics Calculation of Heat Conduction in Liquid and through Liquid-Gas Interface,” Molecular Physics, Vol. 81, pp. 251-261.

Inoue, R., Tanaka, H. and Nakanishi, K., 1996, "Molecular Dynamics Simulation Study of the Anomalous Thermal Conductivity of Clathrate Hydrates," Journal of Chemical Physics, Vol. 104, pp. 9569-9577.

Irving, J. H. and Kirkwood, J. G., 1950, "The Statistical Mechanical Theory of Transport Processes. IV. The Equations of Hydrodynamics,” Journal of Chemical Physics, Vol. 18, pp. 817-829.

Julian, C. L., 1965, "Theory of Heat Conduction in Rare-Gas Crystals,” Physical Review, Vol. 137, pp. A128-A137.

Keyes, R. W., 1959, "Laws of Corresponding States for the Thermal Conductivity of Molecular Solids," Journal of Chemical Physics, Vol. 31, pp. 452-454.

Khare, R., de Pablo, J. and Yethiraj, A., 1997, "Molecular Simulation and Continuum Mechanics Study of Simple Fluids in NonIsothermal Planar Couette Flows,” Journal of Chemical Physics, Vol. 107, pp. 2589-2596.

Klitsner, T., VanCleve, J. E., Fischer, H. E. and Pohl, R. O., 1988, "Phonon Radiative Heat Transfer and Surface Scattering," Physical Review B, Vol. 38, pp. 7576-7594.

Kotake, S., 1994, "Future Aspects of Molecular Heat and Mass Transfer Studies," Microscale and Molecular Heat Transfer, S. Kotake and C. L. Tien, eds., Begell House, Inc., New York, pp. 12-20.

Kotake, S. and Wakuri, S., 1994, "Molecular Dynamics Study of Heat Conduction in Solid Materials," JSME International Journal, Series B, Vol. 37, pp. 103-108.

Kristensen, W. D., Jensen, E. J. and Cotterill, R. M. J., 1974, "Thermodynamics of Small Clusters of Atoms: A Molecular Dynamics Simulation,” Journal of Chemical Physics, Vol. 60, pp. 4161-4169.

Loong, C.-K., Vashishta, P., Kalia, R. K. and Ebbsjo, I., 1995, "Crystal Structure and Phonon Density of States of High-Temperature Ceramic Silicon Nitride,” Europhysics Letters, Vol. 31, pp. 201-206.

Majumdar, A., 1993, "Microscale Heat Conduction in Dielectric Thin Films,” Journal of Heat Transfer, Vol. 115, pp. 7-16.

Massobrio, C. and Ciccotti, G., 1984, "Lennard-Jones Triple-Point Conductivity via Weak External Fields,” Physical Review A, Vol. 30, pp. 3191-3197.

Matsumoto, T., Daimon, M., Mimura, H., Kanemitsu, Y. and Koshida, N., 1995, "Optically Induced Absorption in Porous Silicon and Its Application to Logic Gates," Journal of the Electrochemical Society, Vol. 142, pp. 3528-3533.

Mehta, C. L., 1963, "Coherence-Time and Effective Bandwidth of Blackbody Radiation,” Il Nuovo Cimento, Vol. 28, pp. 1947-1954.

Müller-Plathe, F., 1997, “A Simple Nonequilibrium Molecular Dynamics Method for Calculating the Thermal Conductivity," Journal of Chemical Physics, Vol. 106, pp. 6082-6085.

Novotny, V. and Meincke, P. P. M., 1973, "Thermodynamic Lattice and Electronic Properties of Small Particles," Physical Review $B$, Vol. 8, pp. 4186-4199.

Peierls, R., 1929, "Zur kinetischen Theorie der Wärmeleitung in Kristallen,” Annalen der Physik, Vol. 3, pp. 1055-1101.

Rahman, A., 1964, "Correlations in the Motion of Liquid Argon," Physical Review, Vol. 136, pp. A405-A411. 
Ryckaert, J.-P. and Bellemans, A., 1975, "Molecular Dynamics of Liquid n-Butane Near Its Boiling Point,” Chemical Physics Letters, Vol. 30, pp. 123-5.

Shibahara, M. and Kotake, S., 1998, "Quantum Molecular Dynamics Study of Light-to-Heat Absorption Mechanism in Atomic Systems," International Journal of Heat and Mass Transfer, Vol. 41, pp. 839-849.

Swope, W. C., Andersen, H. C., Berens, P. H. and Wilson, K. R., 1982, "A Computer Simulation Method for the Calculation of Equilibrium Constants for the Formation of Physical Clusters of Molecules: Application to Small Water Clusters," Journal of Chemical Physics, Vol. 76, pp. 637-649.

Tenenbaum, A., Ciccotti, G. and Gallico, R., 1982, "Stationary Nonequilibrium States by Molecular Dynamics," Physical Review A, Vol. 25, pp. 2778-2787.

Tersoff, J., 1988, "New Empirical Approach for the Structure and Energy of Covalent Systems,” Physical Review B, Vol. 37, pp. 69917000 .

Verlet, L., 1967, "Computer "Experiments" on Classical Fluids. I. Thermodynamical Properties of Lennard-Jones Molecules,” Physical Review, Vol. 159, pp. 98-103.

Volz, S. and Chen, G., 1998, "Molecular Dynamics Simulation of Thermal Conductivity in Bulk Silicon and Nanowires," to be presented at the 1998 International Mechanical Engineering Congress and Exposition, Anaheim, California, November 15-20.

Volz, S., Saulnier, J.-B., Lallemand, M., Perrin, B., Depondt, P. and Mareschal, M., 1996, "Transient Fourier-law Deviation by Molecular Dynamics in Solid Argon,” Physical Review B, Vol. 54, pp. 340-347.

White, G. K. and Woods, S. B., 1958, "Thermal Conductivity of the Solidified Inert Gases: Argon, Neon, and Krypton,” Philosophical Magazine, Vol. 3, pp. 785-797.

Wolf, D. and Merkle, K. L., 1992, "Correlation Between the Structure and Energy of Grain Boundaries in Metals," Materials Interfaces: Atomic-Level Structure and Properties, D. Wolf and S. Yip, eds., Chapman and Hall, London, pp. 87-150.

Wolf, D., Wang, J., Phillpot, S. R. and Gleiter, H., 1995, "PhononInduced Anomalous Specific Heat of a Nanocrystalline Model Material by Computer Simulation,” Physical Review Letters, Vol. 74, pp. 4686-4689.

Ziman, J. M., 1960, Electrons and Phonons, Oxford University Press, London. 